\title{
Gamification no Ensino de Funções Administrativas
}

Gesinaldo Santos

André Koscianski

\section{Resumo}

Gamification é um termo surgido há poucas décadas, que se refere à modificar atividades de ensino-aprendizado por meio da introdução de elementos que são próprios de jogos. Essa estratégia busca tratar aspectos como perda de interesse dos alunos, baixa motivação e pouca participação na aula. As modificações efetuadas em sala procuram dar um caráter lúdico às atividades, sem perder o foco nos objetivos didáticos. Existe uma literatura crescente sobre o assunto, mas ainda faltam exemplos de aplicação, sobretudo porque a adaptação de uma atividade depende de conteúdo e público alvo. Este artigo apresenta uma visão geral sobre o assunto e um modelo de gamification disponível na literatura, que pode ser aplicado a diferentes situações. Esse modelo foi empregado na disciplina de teoria da administração em um curso superior; é apresentado todo o planejamento da atividade e sua realização, bem como os resultados gerais e impressões manifestadas pelos alunos.

Palavras-chave: Gamification, Motivação, Disciplina de Teoria da Administração.

\section{Abstract}

\section{Gamification in Teaching Administrative Functions}

Gamification is a term that emerged a few decades, which refers to the teaching-learning activities change through the introduction of elements that are specific to games. This strategy seeks to address issues such as loss of interest of pupils, low motivation and little participation in class. The modifications made in the room looking for a playful character to activities, without losing the focus on didactic objectives. There is a growing literature on the subject, but still lack application examples, particularly because the adaptation of an activity depends on content and target audience. This article presents an overview of the subject and a model of gamification available in the literature, which can be applied to different situations. This model was employed in the discipline of Administration theory in College; are all the planning of the activity and its realization, as well as the General results and impressions expressed by students.

Keywords: Gamification, Motivation, Discipline of Theory of Administration. 


\section{Introdução}

Um grande desafio para todo docente é despertar interesse e motivação nos alunos, mantendo a atenção da turma na aula. Uma simples distração como uma conversa paralela, um toque de celular ou um barulho externo, pode perturbar a concentração de um aluno e eventualmente ocupar o centro da atenção da sala de aula, deixando o conteúdo explicado em segundo plano.

A falta de concentração da sala pode acontecer por diferentes razões. Assuntos mais abstratos e formais, como Matemática e prova de teoremas, tendem a exigir um esforço maior do que conteúdos como Geografia, que guardam relação mais tangível com a realidade. A maneira como o professor expõe um determinado assunto também exerce um efeito determinante sobre o clima em sala de aula e as reações dos estudantes. A rotina diária dos alunos pode ser outra fonte de problemas; indivíduos que tem uma rotina cansativa de trabalho e estudam em contra turno tem mais dificuldade para acompanhar as atividades.

Uma característica fundamental de um jogo é a participação espontânea das pessoas. Dependendo da situação, os jogadores se mantem concentrados mesmo com perturbações externas. Podem agir de maneira autônoma e com iniciativa, além de apresentarem mais vontade de vencer desafios. Um cenário assim é o esperado por qualquer professor, em que alunos apáticos se transformam em participativos, deixando de serem meros personagens do processo de ensino, para se tornarem também protagonistas.

A técnica de Gamification surge como uma alternativa para tratar os problemas mencionados. Elementos presente nos jogos podem ser usados em prol do ensino, para motivar os alunos, e permitir aprender de forma divertida, jogando.

Este artigo discute o uso de gamification no ensino, apresentando uma visão geral a respeito. Tomando como base a abordagem de Huang e Soman (2013), foi estruturada uma atividade lúdica, e para analisar a aprendizagem cognitiva no jogo, foi utilizada a abordagem de Garris, Ahlers e Driskell (2002). O contexto foi uma disciplina de teoria geral da administração de um curso superior de Sistemas de Informação. Originalmente o conteúdo tinha um caráter teórico e era visto como pouco atrativo pelos estudantes; este trabalho analisa a aplicação da estratégia de gamification neste caso. Além de buscar a participação dos alunos, o trabalho também visou passar de uma abordagem essencialmente teórica para a aplicação de conteúdos de maneira dinâmica, dentro da sala de aula.

\section{0 que é Gamification?}

Lazer, e jogos em particular, é parte integral de nossas vidas. O espaço ocupado por essas atividades pode diminuir com o avanço de responsabilidades da vida adulta, mas jamais se anula 
totalmente. Em realidade os jogos mudam e surgem em formas e momentos diferentes, existindo uma ampla literatura que investiga esse assunto (HUIZINGA, 1955, BROUGÈRE, 1995).

Tornar uma atividade maçante em algo aceitável e até mesmo prazeroso é o objetivo central do conceito de Gamification. Há um papel importante para emoções positivas sobre a predisposição de uma pessoa para uma atividade, que pode repercutir sobre o aprendizado (AUSUBEL, 2003).

Para apresentar um exemplo introdutório, pode-se considerar uma aula de revisão de conteúdo, baseada no preenchimento de um questionário ou uma lista de exercícios extensa. Essa tarefa coloca a sala em silêncio, mas nem todos os alunos conseguem sustentar uma concentração intensa, respondendo por longos períodos. A mesma atividade pode continuar a ser realizada, com o mesmo propósito, mas com a atribuição de um prêmio aos primeiros alunos que a concluam. É importante notar que o binômio atividade $\mathrm{x}$ recompensa neste exemplo é apenas parte da situação em sala, não significando que o professor adote uma abordagem behaviorista. Todos os elementos - a competição entre alunos, a maneira de apresentar a tarefa e envolvê-los na realização - fazem parte do projeto instrucional. Ainda neste exemplo, o professor dispõe de várias alternativas: poderia aplicar exercícios sem solução fechada, ou trabalhar com equipes, permitir ou não consultas a internet, etc. Isto ilustra como o uso de gamification se estende a várias situações didáticas.

Gamification pode ser definido como o uso de elementos de jogos, em ambientes que originalmente não tinham esse objetivo (DETERDING et al., 2011, p.2). No ensino, procura-se a inclusão de mecanismos de jogos alinhados com o envolvimento das pessoas na aprendizagem e solução de problemas.

"Gamification should be thought of as the concept of using game-based mechanics, aesthetics and game thinking to engage people, motivate action, promote learning, and solve problems (KAPP, 2012, p.2)."

Assim, o gamification utiliza a estrutura dos jogos para envolver e engajar os alunos na aprendizagem de conteúdos, possibilitando a criação de um ambiente divertido.

Em resumo, o que se procura é tornar o jogo aliado do ensino, empregando toda sua atratividade para que os alunos espontaneamente se esforcem a participar de uma tarefa. Entre os benefícios esperados estão uma melhora momentânea no bem-estar, uma mudança de predisposição em relação ao ambiente da escola e, evidentemente, efeitos positivos no aprendizado e retenção de informações. 


\section{0 que é Gamification?}

O abandono e baixa no desempenho de aprendizagem são reflexos da falta de engajamento e até mesmo de absenteísmo, a ponto de alunos ficarem indispostos com a volta as aulas, e se distraírem com a tecnologia, como smartphones e Internet.

"In education, the reasons for drop-outs or low performance include boredom or lack of engagement, a pattern of escalating absenteeism where each absence makes the person less willing to return to school, and most importantly, being distracted by technology such as smartphones and the Internet (Huang e Soman 2013, p. 5)"

Neste caso a distração pode ser uma tentativa de fuga de um ambiente entediante, para se concentrar em algo que é mais interessante naquele instante. $O$ exemplo clássico disto é deixar de acompanhar uma explicação em sala e consultar redes sociais no celular.

Alinhar a diversão, desafio e competição, dentro de uma atividade em sala sala de aula, pode transportar os alunos e fazê-los sentir como se estivessem em um ambiente de jogo. O clima resultante de gamification é algo ao que estão habituados fora do ambiente da escola. 0 que era antes uma forma de fuga torna-se um mecanismo usado pelo professor para melhorar o engajamento da turma.

Semelhante aos jogos em geral, o uso de gamification em sala de aula abre o uso de regras, surpresas e mesmo sorte, oferece a oportunidade de experimentar emoções e papéis sociais. É possível dividir atividades em fases que correspondem a recompensas. Por exemplo: Quem ler o livro do conteúdo ensinado em sala de aula ganhará pontos de leitura; Quem fizer as atividades de casa durante um mês, ganhará um crachá; Quem quer ser o detetive de ciências? Ao jogar com essas regras, os estudantes desenvolvem novos quadros para compreensão das atividades escolares (LEE, HAMMER, 2011, p.2)

A motivação com o uso da diversão está associada a vários elementos interessantes para a sala de aula, como autonomia, competência e conexão com outros participantes (RYAN, et al., 2006, p.1).

Outro ponto importante, é que o gamification permite aprender por meio da repetição, uma estratégia que, se empregada de forma isolada, remete a ideia de algo cansativo. Em jogos a repetição acontece naturalmente para conseguir passar de fases.

Os jogos podem promover bons resultados na aprendizagem, visto que no jogo o aluno é estimulado a aplicar conhecimento aprendido, bem como desenvolver a percepção de aplicação desses conhecimentos em diferentes contextos (GEE, 2003). $O$ ensino com jogos pode envolver conhecimento em diferentes categorias (GARRIS et al, 2002, p.456):

148 DOI: Em andamento. 
O Conhecimento Declarativo refere-se ao conhecimento dos fatos e dados necessários para execução da tarefa.

O Conhecimento Processual refere-se a como executar uma tarefa, bem como demonstrar a capacidade de aplicar conhecimento ou habilidades para uso específico.

O Conhecimento Estratégico refere-se a aplicação de princípios aprendidos em diferentes situações, no qual desenvolve a compreensão quando e por que os princípios aplicam-se.

Os Resultados da Aprendizagem Afetiva referem-se ao conjunto de confiança, auto eficácia, atitudes, preferências e disposições, que são sentimentos e um tipo de específico de aprendizagem, no qual a mudança de atitude é um objetivo na formação da construção.

\section{Como Aplicar Gamification no Ensino?}

A aplicação de gamification deve ser planejada; isso requer antecipar o funcionamento da atividade em sala, do contrário, pode passar de uma estratégia de ensino, com boas possibilidades de aprendizagem, para uma mera "brincadeira", em que alunos observam apenas como uma distração, sem foco educativo.

Um modelo para criar o projeto instrucional é proposto por Huang e Soman (2013), conforme "Figura 1", dividindo o planejamento em cinco etapas.

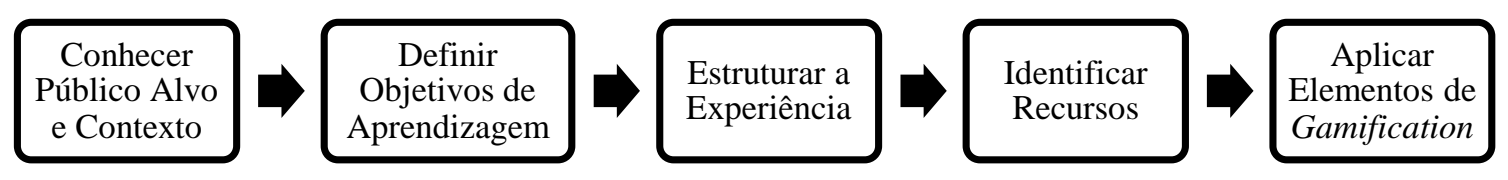

Figura 1: Etapas para aplicar Gamification

(HUANG e SOMAN, 2013)

As cinco etapas do processo acontecem de maneira sequencial, como ilustrado na Figura

1. Elas são descritas a seguir.

Etapa 1 - Compreender o Público Alvo e o Contexto:

Conhecer o aluno e compreendê-lo é um ponto chave para a experiência de gamification, pois ao analisar o público alvo, obtêm-se informações como a faixa etária, habilidades atuais e habilidades pretendidas de aprendizagem. E ao analisar o contexto, obtêm-se informações como o ambiente de aprendizagem, tamanho do grupo e sequenciamento das habilidades.

Com base nessas informações, a técnica de gamification poderá ser aplicada de forma direcionada para a aprendizagem, ou seja, no local mais adequado, na duração mais aceitável, se será em equipe ou individual, quais as habilidades necessárias, dentre outros.

DOI: Em andamento. 
Dessa forma, é importante considerar a personalização da atividade para cada tipo de público, respeitando seus limites e seus conhecimentos, pois ao criar um padrão, fundamentado em uma classe específica, não se considera a diversidade dos alunos, bem como suas peculiaridades, fatores extremamente relevantes.

\section{Etapa 2-Definir os Objetivos de Aprendizagem}

Ao definir os objetivos de aprendizagem o professor também seleciona os materiais a serem usados e a estratégia a ser empregada. No exemplo comentado na seção 2 , a aplicação de um questionário poderia ser o método escolhido pelo professor para revisar um conteúdo. Outra possibilidade seria elaborar uma apresentação em sala, por equipes; ou criar um texto colaborativamente. As escolhas que o professor faz, nesse momento, dependem basicamente de como prefere abordar um dado objetivo em sua sala de aula. A ideia de gamification virá depois revestir a atividade e eventualmente mudar algumas características e forma de aplicação, mas, em essência, a atividade em si e os objetivos de aprendizagem permanecem sendo definidos exclusivamente segundo critérios de ensino.

\section{Etapa 3 - Estruturar a Experiência:}

Elaborar etapas e marcos é importante para indicar ao professor, o andamento da atividade. Além disso, sendo possível dividir uma tarefa em etapas, cria-se a oportunidade de introduzir elementos adicionais.

Ao controlar etapas busca-se conhecer a absorção de conhecimento dos alunos, qual a sequência de apresentação mais adequada, ou como retroagir ou avançar, quando necessário. Logo, o professor dispõe de indicadores que demonstram a real situação da atividade por etapa, e tem fundamentos para decisões em cada etapa, não correndo o risco de observar problemas somente no final.

Também é importante determinar objetivos diferentes para cada etapa. É recomendável começar com etapas fáceis, que motivem e comprometam o aluno.

Etapa 4-Identificar os recursos:

Depois da identificação das etapas e marcos, o professor deve julgar quais poderão utilizar o gamification. Para tanto, é preciso considerar alguns pontos:

Mecanismo de Rastreamento: possibilita medir o nível de progresso de aprendizagem do aluno, bem como identificar dificuldades encontradas.

Moeda: é uma unidade de medição que pode ser pontos, tempo, entre outros.

Nível: uma quantidade específica de uma moeda para realizar um objetivo.

Regras: são os limites que o aluno pode ou não pode fazer na atividade.

150 DOI: Em andamento.

R. Bras. de Ensino de C\&T 
Feedback: é um mecanismo que pode ser usado, tanto pelo professor, quanto o aluno, uma vez que demonstra o progresso de aprendizagem, que pode ser positivo ou negativo da atividade.

Como exemplo desses itens, pode-se considerar a aprendizagem de multiplicação em Matemática. A atividade poderia ser feita em um prazo estipulado, assim, o tempo é a unidade de medição (moeda); os que executaram em menor tempo dispõem de maior conhecimento (mecanismo de rastreamento); para que se passe de fase seria preciso obter um tempo inferior aos estipulado (definição de níveis); mas além de terminar nesse tempo, o aluno deveria também explicar como chegou ao resultado (regra); e a cada etapa o professor corrigiria os possíveis erros (feedback).

\section{Etapa 5 - Aplicar Elementos próprios de Gamification}

Os elementos de jogos podem ser divididos em dois tipos: básicos e sociais.

Os elementos básicos incluem pontos, níveis ou simplesmente a restrição do tempo. Esses elementos ajudam o estudante a concentrar-se na atividade, querendo ganhar mais pontos, e passar de fase, pois por meio de uma "recompensa", o interesse e comprometimento dos alunos são despertados.

Os elementos sociais promovem a interação entre os alunos, visto que aos estarem inseridos em uma comunidade, suas ações se tornam públicas e o progresso das atividades é acompanhado por todos participantes.

A escolha dos elementos, demonstrados no Quadro 1, deve ser feita pelo professor de maneira a adequar a atividade aos objetivos que pretende realizar. Por exemplo, para completar uma fase, o aluno precisa adquirir habilidades e competências específicas, mas se a fase for difícil pode promover a desmotivação, e comprometer a aprendizagem. Entretanto, se utilizar um elemento como pontuação intermediária dentro das fases, isso pode promover a melhoria contínua, pois quanto maior a pontuação, maior a realização do aluno naquela tarefa particular.

Quadro 1 - Exemplos de mecanismos de jogos.

\begin{tabular}{|l|l|}
\hline Elementos básicos & Elementos Sociais \\
\hline Pontos & Líderes \\
\hline Níveis & Bens Virtuais \\
\hline Troféus & Cooperação Interativa \\
\hline Bens Virtuais & Linha de História \\
\hline Linha de História & \\
\hline
\end{tabular}

DOI: Em andamento. 


\begin{tabular}{|l|l|}
\hline Restrição de Tempo & \\
\hline Estética & \\
\hline
\end{tabular}

Em resumo, para que haja uma boa aplicação de gamification no ensino, é preciso inicialmente conhecer os alunos, saber de suas limitações e lacunas de aprendizagem (Passo 1). Posteriormente, fundamentado nessas informações, elaborar um objetivo claro (Passo 2), com metas medidas e administradas a cada etapa (Passo 3 ) para que o objetivo seja alcançado. Em sequência, saber qual o recurso de gamification mais adequado para cada etapa (Passo 4), uma vez que por meio desses recursos, consegue-se medir efetivamente cada etapa da atividade, sejam por meio de pontos, feedbacks, regras, entre outros. E finalmente, escolher elementos de gamification (Passo 5), compondo a atividade de acordo com os objetivos desejados.

\section{Metodologia}

O estudo a respeito de gamification embasou uma aplicação feita em sala de aula, no ensino superior. A disciplina trabalhada foi Teoria Geral da Administração, com 42 alunos do primeiro semestre do curso de Sistemas de Informação de uma Faculdade Particular no estado de São Paulo.

O conteúdo tratado foi funções administrativas, que em resumo, são as atribuições que um administrador precisa executar como: o planejamento, a organização, o controle e a liderança.

O professor ficou presente na sala e era o responsável por toda a administração da atividade, desde a organização das equipes, até a soma dos pontos no jogo. A cada etapa, fornecia feedback da teoria sendo aplicada na atividade.

A pesquisa teve uma característica exploratória, com o objetivo principal de aprimoramento de ideias, além de apresentar aspectos da forma de um estudo de caso (GIL, 2002, p.41). Os dados coletados nessa pesquisa incluíram a observação direta e opiniões dos alunos coletadas por escrito, sobre a atividade que executaram. A análise qualitativa visava obter um quadro sobre a receptividade dos alunos, possíveis falhas ao longo do processo e possibilidades de melhoria.

\section{Gamification no Ensino de Funções Administrativas: A Fábrica de Aviões de Papel}

A ideia de utilizar o recurso de gamification originou-se das seguintes perguntas: como empregar a prática em conteúdos que vislumbram a teoria? E como tornar interessante o aprendizado de uma teoria, a princípio maçante?

152 DOI: Em andamento.

R. Bras. de Ensino de C\&T 
Elaborou-se uma atividade que corresponde a uma fábrica de aviões de papel.. Para isso foram seguidas as cinco etapas descritas na seção anterior.

Na primeira etapa buscou-se compreender o público alvo e o contexto. A maioria da sala era formada por homens, contando com apenas 4 mulheres. A faixa etária era entre 18 e 22 anos. Durante as aulas, devido a teoria dos conteúdos ser predominante, a postura dos alunos era bastante apática. Além disso, por ser uma turma do primeiro semestre, o relacionamento entre os alunos ainda era restrito e não havia muito entrosamento para trabalhos em equipe.

A segunda etapa era definir o objetivo da aprendizagem. No presente caso, os estudantes tinham um conhecimento abstrato de funções como planejamento e controle, obtido por um estudo teórico ou, no máximo, pela leitura de exemplos de aplicação. O desafio era definir uma tarefa a ser feita em sala, de caráter lúdico e envolvente, na qual os estudantes aplicassem esses conceitos de administração. A princípio qualquer atividade simples e repetitiva, que pudesse ser feita em grupo, poderia ser adaptada aos objetivos. Assim, escolheu-se uma fábrica de aviões de papel.

A terceira etapa foi a elaboração de marcos e etapas para organizar a tarefa. Foram definidos 3 marcos: a percepção de planejamento; a organização em si; e por fim a análise do controle. Em todos os casos analisou-se também a liderança.

A quarta etapa do processo de projeto instrucional foi identificar os recursos. Para isso pensaram-se em mecanismos de rastreamento, uso de uma moeda virtual, regras e feedback.

$\mathrm{Na}$ quinta etapa identificou-se que elementos próprios de jogos seriam úteis dentro da atividade em sala. Esses elementos ajudariam a enfatizar o lado lúdico e pensou-se nos elementos troféu, contagem de pontos e restrição do tempo. A definição de líderes de equipe também pode ser vista como um elemento social de jogo.

Dinâmica do Jogo

Uma semana antes da aula, o pesquisador enviou aos alunos um e-mail, solicitando que trouxessem 10 folhas A4 (rascunho) cortadas ao meio, para uma atividade em sala de aula.

No dia da atividade e já em sala de aula, o pesquisador comunicou que seria executada uma atividade que mostraria a importância do uso das funções administrativas. Foi mencionado então que se tratava da simulação de uma fábrica de aviões de papel.

O pesquisador solicitou que os alunos se dividissem em equipes de 7 componentes. Cada equipe deveria escolher um nome para a "fábrica". Totalizou-se 6 equipes.

Foram então lidas as regras do jogo:

- o avião precisa ter 6 janelas, 2 portas e o símbolo da equipe em cada asa; 
- as janelas devem ser desenhadas com caneta na cor azul, as portas na cor vermelha e o símbolo na cor preta;

- o ciclo de produção será de 2 minutos;

- o ciclo de planejamento será de 1 minuto;

- a equipe que conseguir produzir mais aviões, vence o jogo.

Logo após a leitura das regras, o pesquisador desenhou uma tabela no quadro-negro. Cada linha representava uma equipe, enquanto nas colunas seriam anotados dados durante a execução da atividade.

Em sequência, iniciou-se o jogo com a primeira pergunta do pesquisador: quantos aviões a fábrica de vocês, consegue produzir em 2 minutos? Nesse momento, já se inicia a percepção de uma função administrativa, o planejamento, visto que os alunos tiveram que pensar e analisar a melhor forma de produzir, além de haver um consenso com os membros da equipe.

Posterior ao planejamento, o pesquisador solicitou que cada equipe falasse a quantidade de aviões que planejava produzir, anotando no quadro-negro os dados. Em seguida iniciou-se a primeira fase do jogo.

As equipes tiveram o tempo de 2 minutos para produzir aviões de papel. Terminado o prazo, realizou-se a contagem do resultado obtido por cada equipe. Os números foram também anotados no quadro-negro. O Quadro 2 mostra esses dados e os números obtidos nas fases seguintes do jogo.

Quadro 2 - Placar com as fases do jogo

\begin{tabular}{|c|c|c|c|c|c|}
\hline Equipe & Planejamento & Fase 1 & Fase 2 & Fase 3 & Total \\
\hline SDMI & 20 & 0 & 6 & 10 & 16 \\
\hline AMMJG & 5 & 0 & 2 & 7 & 9 \\
\hline AGUIA & 20 & 3 & 6 & 6 & 15 \\
\hline OMEGA & 15 & 1 & 1 & 3 & 5 \\
\hline ABJWL & 7 & 4 & 6 & 6 & 16 \\
\hline MARABARROS & 20 & 2 & 5 & 10 & 17 \\
\hline FRACTAL & 30 & 1 & 4 & 7 & 12 \\
\hline
\end{tabular}

Pode-se observar uma grande diferença entre as quantidades planejadas e as realizadas por todas as equipes na primeira fase. 
Durante essa pausa, foram feitos comentários sobre a utilidade das funções administrativas para melhorar a produção. $O$ pesquisador citou a organização dos recursos (humanos - alunos e materiais - canetas e papéis), bem como uma melhor disposição de produção em equipe, ao invés de cada componente fazer o avião de forma individualizada.

Após o feedback e a organização dos recursos, iniciou-se a Fase 2. Novamente os estudantes tiveram um tempo de 2 minutos para produzir aviões de papel, sempre de acordo com as especificações combinadas no início de aula.

Nessa fase, notou-se um aumento na produção, conforme apresentado no "Quadro 2". Todavia, novamente o pesquisador forneceu feedback da etapa. Foi proposto usar outra função administrativa, o controle. As equipes precisavam estipular metas claras (planejamento), organizar os recursos e controlar todo o processo. E também, cada membro da equipe precisava utilizar com maior ênfase outra função administrativa, a liderança, no qual deveria exercer a comunicação e cobrança dos demais membros a participação na fabricação de aviões.

Iniciou-se então a última etapa do jogo, a Fase 3. Ao final, notou-se uma evolução significativa entre as fases, a ponto de a equipe SDMI iniciar a fase com 0 aviões, e ao final chegar a produzir 10 aviões. Por meio da observação sistemática, o pesquisador associou essa evolução com a aplicação das funções administrativas entre as fases do jogo, no qual a equipe vencedora usou o planejamento para analisar o que poderia ser melhorado a cada fase; a organização com a melhor disposição dos papéis a serem dobrados e desenhados pelos membros da equipe; o controle para alcançar e ultrapassar a meta proposta na produção de aviões, e finalmente a liderança no incentivo e motivação dos membros da equipe.

\section{Resultados e Discussões}

Como forma de ilustrar a contribuição positiva de uma atividade de gamification aplicada ao ensino de das funções administrativas, segue abaixo algumas respostas dos alunos para a seguinte pergunta: "O que vocês acharam da atividade? Foi algo positivo, negativo? Explique". O pesquisador salientou que a resposta seria anônima.

A figura 2 demonstra o conhecimento processual, no qual os alunos puderam demonstrar e aplicar conhecimento e o conhecimento estratégico, com a compreensão dos princípios que aplicaram (GARRIS, et al., 2002, p.456). Também, aparece a percepção de análise da situação, escolha da teoria aprendida e aplicação, objetivando a melhoria contínua (JOHNSSON, 2006, p.39). Dessa forma, os alunos aplicaram na prática, os princípios de administração, normalmente vistos de forma muito teórica no curso. 


\section{For muito produtivo, pass, purcebucemos so plonefar a sogamizar datermos melhores resultados,}

Figura 2 - Resposta dos alunos

A figura 3 demonstra o conhecimento processual com a execução da tarefa por meio da teoria; conhecimento estratégico com a aplicação dos conhecimentos aprendidos; e aprendizagem afetiva com o conjunto de confiança para melhoria futura (GARRIS, et al., 2002, p.456). Assim, semelhante a resposta anterior, os alunos assimilaram a relevância dos processos administrativos, a ponto de identificarem pontos de melhoria e aplicarem correções, semelhante a uma situação profissional real. Esse é um efeito previsto do uso de jogos no contexto de simulação de tarefas reais (GEE, 2003). Os participantes também assimilaram a prática de ações de planejamento e tomada de decisão como melhoria nos resultados (MARTINELLI, 1988, p.26). Outro ponto relevante foi que os próprios alunos visualizaram a atividade de gamification, não apenas como um jogo, mas como um método para estudar.

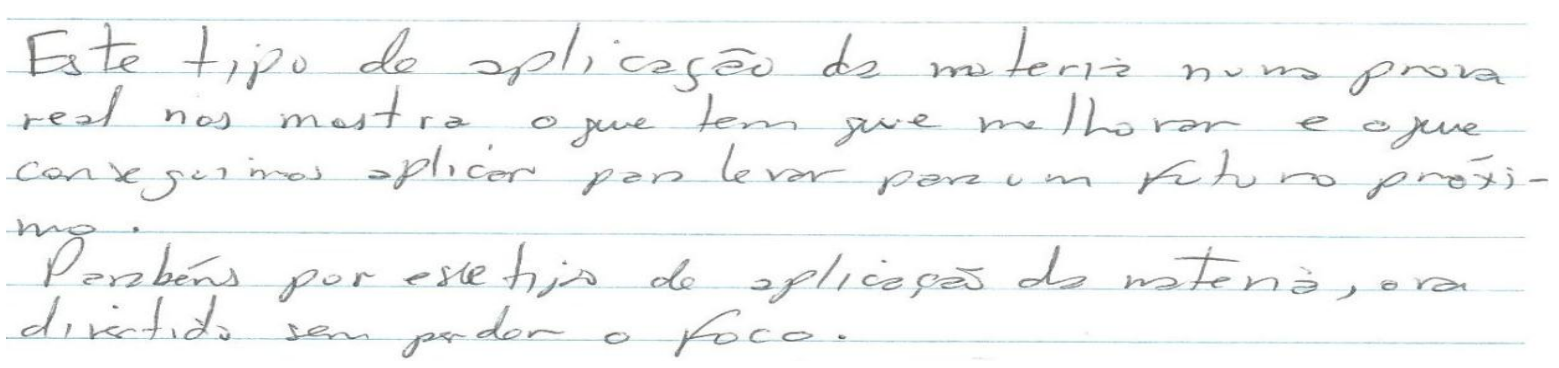

Figura 3 - Resposta dos alunos

$\mathrm{Na}$ figura 4 os alunos demonstraram a aprendizagem afetiva, com o conjunto de confiança na atividade, bem como preferências e disposições para atividades lúdicas similares (GARRIS, et al., 2002, p.456). Houve o desenvolvimento da habilidade de interação com os demais participantes, através do jogo (FREITAS, 2007, p.36), inclusive com a menção de ajuda na cooperação e convivência. E no elogio do método de trabalho, visto que nesse método o aprendizado dos participantes não é somente pela observação, mas também pela vivência prática, jogando e planejamento, por meio do aprendizado de soluções de problemas (NIVEROS, 2004, p.97). Nessa resposta, os alunos se identificaram, mesmo com o pesquisador enfatizando a resposta anônima, e gostaram do método de trabalho. 


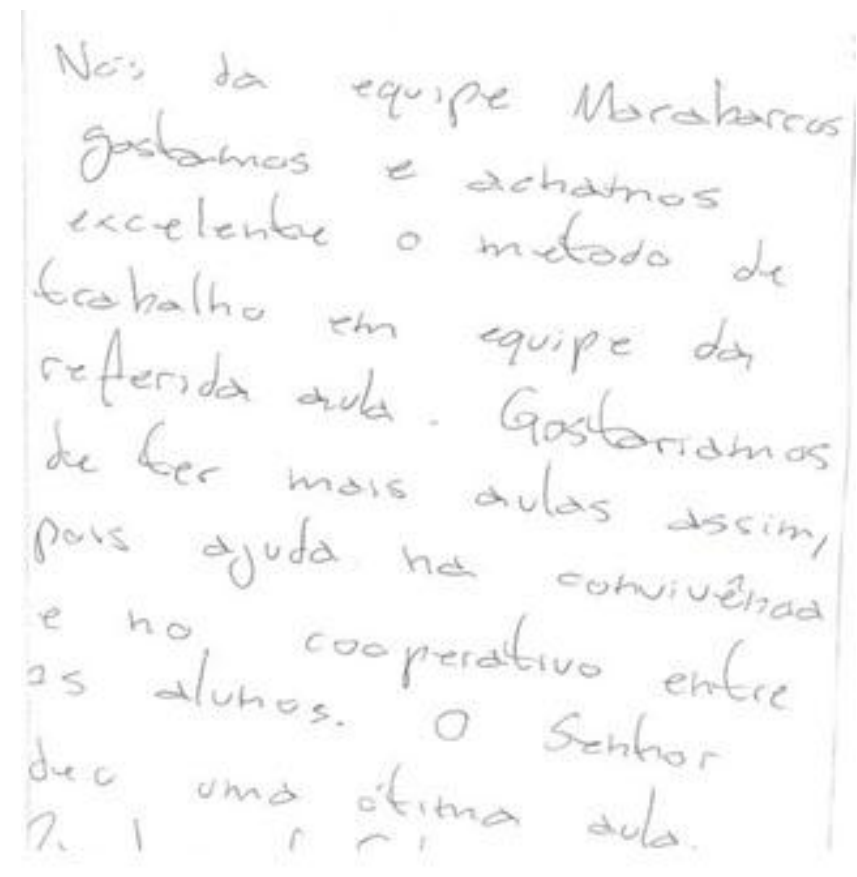

Figura 4 - Resposta dos alunos

A atividade demonstrou que um conteúdo, originalmente tratado de maneira bastante teórica, pode se tornar atrativo e interessante para o professor e alunos. Ao longo da aula, observou-se nos alunos a alegria na realização das etapas do jogo e o entrosamento entre eles, o que era um resultado apontado pela literatura (RYAN, et al., 2006). Observou-se também um processo de comunicação individual e em equipe, que exigiu habilidade de ouvir, processar, entender e repassar informações; respeitar pontos de vista; cooperar com os colegas e tratar ideias de forma flexível apontado pela literatura (GRAMIGNA, 1993).

Assim, alunos originalmente introvertidos se mostraram mais participantes e se relacionaram com os demais membros da equipe. Para o pesquisador que anteriormente ministrava esse mesmo conteúdo somente de forma teórica, os feedbacks durante a atividade foram importantes, pois o conteúdo foi revisado e ilustrado com a aplicação da teoria estudada.

\section{Considerações Finais}

De maneira geral, quando os conteúdos de aprendizagem se tornam mais complexos e o ensino mais exigente, aumentam as possibilidades da sala de aula ser percebida pelos alunos como pesada, séria ou estressante. As estratégias de gamification não alteram o conteúdo sendo tratado em sala, e por vezes não alteram sequer os instrumentos usados - como trabalhos, questionários, apresentações. Pode-se modificar o cenário da sala de aula em que as atividades acontecem, incluindo-se regras e outros elementos retirados de jogos, de maneira a alterar a participação dos alunos e torná-la mais agradável.

DOI: Em andamento. 
Essa mudança de participação pode ser aproveitada para que o aluno se envolva com o conteúdo de uma forma ativa. Além do exemplo apresentado neste trabalho, podem-se derivar outras possibilidades usando a metodologia discutida, de Huang e Soman (2003). Alunos podem trocar entre si explicações de matemática para obter pontos num jogo. Equipes podem trocar desafios na forma de perguntas de geografia. Uma classe de história pode usar a organização de um RPG (role-playing-game) como método para abordar um conteúdo.

Nesse estudo, gamification foi aplicado em uma disciplina de fundo teórico, a calouros de ensino superior. Isso propiciou o envolvimento ativo dos alunos com os conteúdos estudados. Além do objetivo principal, de evitar uma aula entediante, foi possível levar a classe a vivenciar em pequena escala a aplicação de conceitos de administração. Finalmente, em uma turma de alunos que se conheciam depois de pouco tempo, houve muita comunicação durante a atividade e um excelente entrosamento.

\section{Referências}

AUSUBEL, D. P. Aquisição e retenção de conhecimentos: uma perspectiva cognitiva. 1. ed. Lisboa: Plátano Edições Técnicas, 2003.

BROUGÈRE, G. Jeu et éducation. Editions L'Harmattan, 1995.

DETERDING, S.; DIXON, D.; KHALED, R.; NACKE, L. From Game Design Elements to Gamefulness: Defining “Gamification". MindTrek'11, September 28-30, 2011, Tampere, Finland

FREITAS, C. C. G.. Aprendizagem Experimental e Jogos de Empresas no Estudo do Mercado de Capitais: uma Aplicação. 2007. Dissertação (Mestrado em Administração) - Universidade Estadual de Londrina e Universidade Estadual de Maringá.

GARRIS, R.; AHLERS, R.; DRISKELL, J.. Games, Motivation, and Learning: A Research and Practice Model. SIMULATION \& GAMING, Vol. 33 No. 4, December 2002 441-467

GEE, J. P. What video games have to teach us about learning and literacy. New York: Palgrave Macmillan, 2003.

GRAMIGNA, M. R. M., Jogos de Empresa, São Paulo, Makron Books, 1993.

HUANG, W. Y.; SOMAN, D. A Practitioner's Guide To Gamification Of Education. Rotman School of Management, University of Toronto, Dez/2013.

HUIZINGA, J. Homo Ludens: A Study of the Play-element in Culture. Beacon Press, 1955.

JOHNSON, M. E.. Jogos de Empresas: Modelo para Identificação e Análise de Percepções da Prática de Habilidades Gerenciais. 2006. Tese (Doutorado) - Universidade Federal de Santa Catarina. 
KAPP, K. M.; CONÉ, J.. What Every Chief Learning Officer Needs to Know about Games and Gamification for Learning. Department of Instructional Technology and Institute for Interactive Technologies, 2012.

LEE, J. J.; HAMMER, J. Gamification in Education: What, How, Why Brother? Academic Exchange Quarterly Disponível em: http://www.academia.edu/570970/Gamification_in_Education_What_How_Why_Bother Acesso em: $14 / 03 / 2014$

MARTINELLI, D. P.. A Utilização dos Jogos de Empresa no Ensino da Administração. Revista de Administração, São Paulo 23 (3):24-37, junho/setembro 1988.

NIVEROS, S. I. As Possibilidades da Tecnologia no Auxílio da Aprendizagem através dos Jogos Educacionais. 2004. Tese (Doutorado em Engenharia da Produção) - Universidade Federal de Santa Catarina, Florianópolis.

RYAN, R. M.; RIGBY, C. S..; PRZYBYLSKI, A.. The Motivational Pull of Video Games: A SelfDetermination Theory Approach. Springer Science+Business Media, LLC, 2006.

Gesinaldo Santos. Mestre em Ensino de Ciência e Tecnologia pela Universidade Tecnológica Federal do Paraná (UTFPR), Especialista em Gestão da TI pela Faculdade de Tecnologia (FATEC); Especialista em Gestão Estratégica de Negócios pela Universidade do Centro do Paraná (UCP); Licenciado em Matemática pela Faculdade Paulista São José; Graduado em Administração de Empresas pela Faculdades Integradas de Itararé (FAFIT). Docente das Faculdades Integradas de Itararé nos cursos de Sistemas de Informação e Administração de Empresas.

André Koscianski. Graduado em Bacharelado Em Ciência da Computação pela Universidade Estadual de Maringá (1993), mestrado em Engenharia Elétrica e Informática Industrial pela Universidade Tecnológica Federal do Paraná (2000) e doutorado em Simulação - Institut National Des Sciences Appliquées de Rouen (2004). Professor da Universidade Tecnológica Federal do Paraná, atuando na graduação e no programa de mestrado PPGECT. Trabalhos em torno de jogos e multimídia voltando-se atualmente à aplicações em ensino no mestrado. 